\title{
Domain Transformation of Chest CT Images Using Semi-Supervised Cycle GAN for Opacity Classification of Diffuse Lung Diseases
}

\author{
Masashi Miyake \\ Graduate School of Sciences and Technology for Innovation, Yamaguchi University, Tokiwadai2-16-1 \\ Ube, Yamaguchi 755-8611, Japan \\ Shingo Mabu \\ Graduate School of Sciences and Technology for Innovation, Yamaguchi University, Tokiwadai2-16-1 \\ Ube, Yamaguchi 755-8611, Japan \\ Shoji Kido \\ Graduate School of Medicine, Osaka University, Yamadaoka2-15 \\ Suita, Osaka 565-0871, Japan \\ Takashi Kuremoto \\ Graduate School of Sciences and Technology for Innovation, Yamaguchi University, Tokiwadai2-16-1 \\ Ube, Yamaguchi 755-8611, Japan
}

\begin{abstract}
The aim of this research is to perform domain translation of chest CT images so that medical institutions can effectively use a computer-aided diagnosis (CAD) system trained at a different institution. We propose a semisupervised Cycle GAN for domain transformation by combining the standard Cycle GAN and the trained CAD. In the experiment, we classified opacities of diffuse lung diseases in CT images and clarified the effectiveness of the proposed method for domain transformation.
\end{abstract}

Keywords: Deep Learning, Domain transformation, GAN, chest CT, Classification, Semi-supervised

\section{Introduction}

Recently, with the development of digital medical imaging devices, it has become possible to acquire an enormous amount of medical images. Therefore, studies on computer-aided diagnosis (CAD) have been actively conducted, where machine learning is applied to build CAD systems using a large number of medical images. Image processing and image recognition techniques are used to construct CAD systems. Recently, the research of convolutional neural networks has been rapidly developed in the research field of image recognition and has been applied to classifiers used in CAD systems ${ }^{1}$. However, training a classifier requires a large amount of annotated medical image data. Also, there are possibilities that the accuracy of diagnosis may fluctuate when the photography conditions of the images are different depending on the medical institutions. For example, different CT systems and their settings can change the pixel values. Therefore, a CAD system showing good diagnostic performance in a certain medical institution does not always show the same performance in other medical institutions. In that case, the classifier needs to be retrained, and a large amount of training data for each medical institution needs to be prepared for training. The current system is not easy to be used widely in various medical institutions, and it is necessary to solve this problem. 


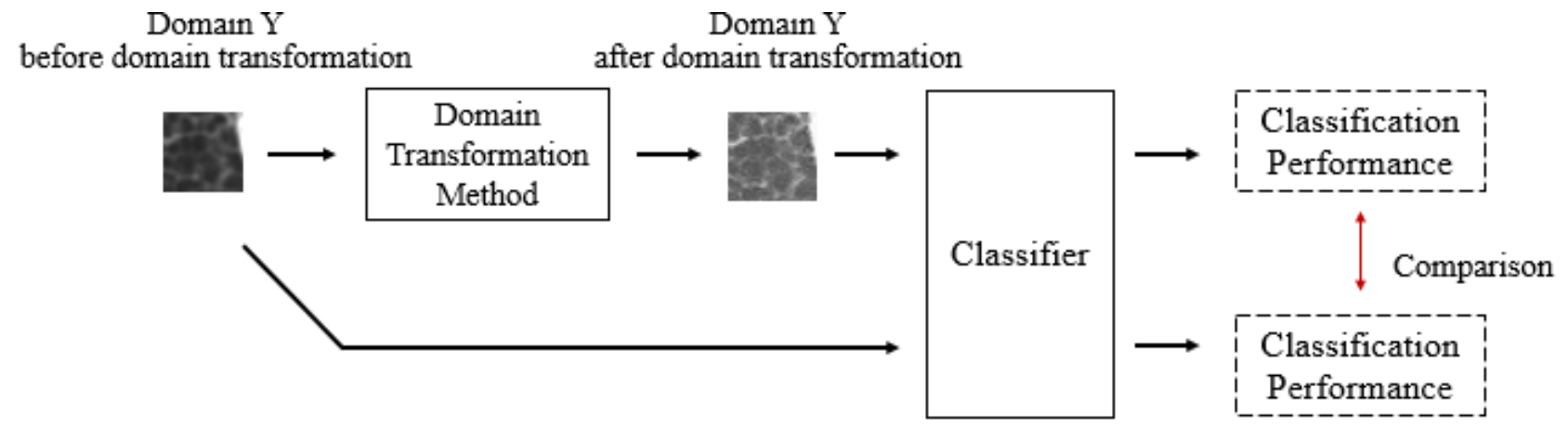

Fig. 1. The flow of the validation of the proposed method. We compare the classification performance of several domain transformation methods including the proposed method and without domain transformation. Refer to Table 1 for the details of domain transformation methods.

A domain transform method to classify opacities in chest $\mathrm{CT}$ images using Cycle $\mathrm{GAN}^{2}$ has been proposed as a method to solve the above problem. However, since the training of domain transformation does not use labeled data, the transform is not always suitable for the classification of the lung opacities. Hence, we propose a semi-supervised Cycle GAN that combines the standard Cycle GAN with a trained classifier that is trained in other medical institutions. In detail, 1) the trained classifier classifies a small number of images of another medical institution after the images are domain transformed by Cycle GAN. 2) We examine whether the domain transformation by Cycle GAN is appropriately executed or not. 3) Cycle GAN re-train based on the results. The above 1), 2) and 3) are repeated to make the domain transformation suitable for the opacity classification.

\section{Proposed Method}

This study aims to classify opacities of diffuse lung diseases in chest CT images, where we also aim to diverse a classifier based on the domain transformation of semi-supervised Cycle GAN. In detail, we regard the conditions of the CT images taken at Osaka University Hospital (hereafter referred to as Domain O) as the standard domain and convert the CT images taken at Yamaguchi University Hospital (hereafter referred to as Domain Y) to the conditions of Domain O using semisupervised Cycle GAN. This domain transformation from domain $\mathrm{Y}$ to domain $\mathrm{O}$ is defined as $\mathrm{Y}$ toO transformation.
The semi-supervised Cycle GAN used for YtoO transformation is a structure that combines a standard Cycle GAN with a trained classifier. The structure of the classifier is based on a Residual Network ${ }^{4}$ (ResNet), and the ResNet is trained with domain $\mathrm{O}$ image data. ResNet classifies the opacity of a small number of domain $\mathrm{Y}$ images after the domain transformation YtoO by Cycle GAN has performed. Based on the classification result, Cycle GAN is re-trained.

The flow of the validation of the classification methods is shown in Fig. 1 and the methods for the validation are listed in Table 1 . We measure classification accuracy averaged over 20 independent trials and apply the t-test between the proposed method and the other methods. After the validation in the case of YtoO, OtoY transformation is also verified.

\subsection{Creating ROI Images}

In this study, 503 chest CT images taken at Yamaguchi University Hospital and 636 chest CT images taken at Osaka University Hospital are used. Examples of the chest CT images taken at each hospital are shown in Fig. 2. The chest $\mathrm{CT}$ images of domains $\mathrm{Y}$ and $\mathrm{O}$ contain six opacities: consolidation (CON), diffuse nodular (DN), emphysema (EMP), ground-glass opacity (GGO), honeycombing (HCM), and normal (NOR). The CT images of domains $\mathrm{Y}$ and $\mathrm{O}$ are divided into $32 \times 32$ [pixels] regions of interest (ROIs), which are used for training the Cycle GAN. Each CT image has a mask image that shows the location of each opacity. The mask images were created by three radiologists. $32 \times 32$ [pixels] regions are scanned while striding from the upper left 
Table 1. Condition of each method. We compare four different methods including the proposed method. The architecture of the proposed model is a combination of a standard Cycle GAN and a ResNet trained on the target domain: classifier trained at the other medical institution. "ResNet trained with source domain" represents ResNet trained with only a few teacher labels for semi-supervised learning.

\begin{tabular}{lccc}
\hline & Cycle GAN & \multicolumn{2}{c}{ With ResNet } \\
\cline { 2 - 4 } Domain transformation method & & $\begin{array}{c}\text { Trained with } \\
\text { source domain }\end{array}$ & $\begin{array}{c}\text { Trained with } \\
\text { target domain }\end{array}$ \\
\hline No domain transformation & $\checkmark$ & & \\
Cycle GAN & $\checkmark$ & $\checkmark$ & $\checkmark$ \\
Method for comparison & $\checkmark$ & & $\checkmark$ \\
Semi-supervised Cycle GAN (Proposed method) & $\checkmark$ & & \\
\hline
\end{tabular}
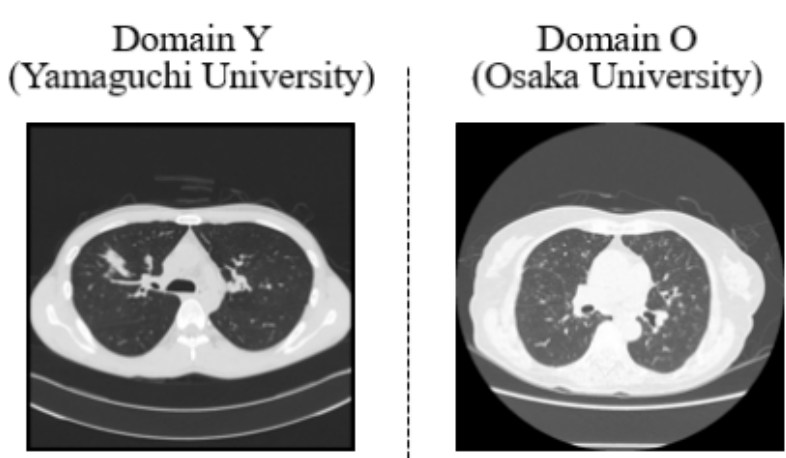

Fig. 2. Examples of chest CT images. The left is a chest CT image taken at Yamaguchi University Hospital, and the right is a one taken at Osaka University Hospital.
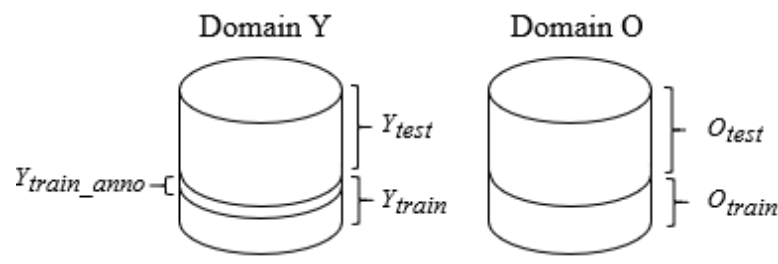

Fig. 3. Training data and testing data of domain $\mathrm{Y}$ and $\mathrm{O}$. The created ROI images are split into a set of training data and test data. $Y_{\text {train }}$ and $O_{\text {train }}$ are used to train Cycle GAN, while $Y_{\text {test }}$ and $O_{\text {test }}$ are used to verify the effectiveness of the domain transformation. $Y_{\text {train_anno }} \subset Y_{\text {train }}$ is a dataset of a small number of $Y_{\text {train }}$ with class labels. Specifically, CT images of five patients are annotated for each opacity.

corner of the mask image to the lower right corner. If the regions contains more than $50 \%$ of the masked area indicating each opacity, the same areas are extracted from the corresponding $\mathrm{CT}$ images and used as ROI images.

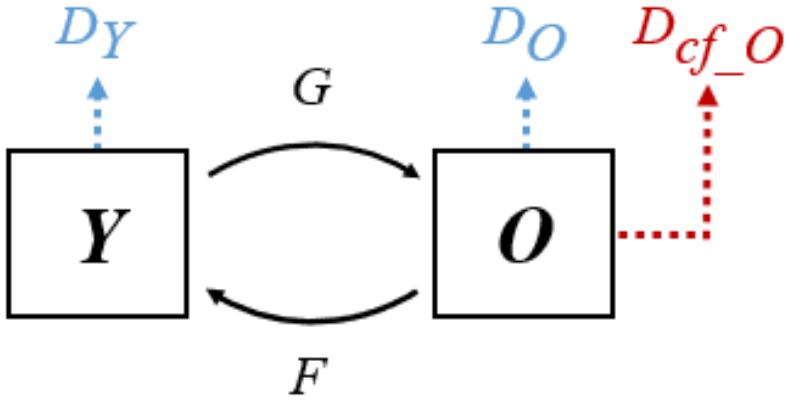

Fig. 4. Structure of semi-supervised Cycle GAN. Semisupervised Cycle GAN contains a standard Cycle GAN consisting of two generators and two discriminators, and also contains a classifier $D_{c f_{-} o}$ that classifies the opacity. Discriminator $D_{o}$, which discriminates real data $\{0\}$ and transformed data $G(y)$, learns to achieve high discrimination accuracy. In other words, $D_{O}$ learns to maximize $\log (1-$ $\left.D_{O}(G(y))\right)$ and $\log D_{O}(o)$. While generator $G$, which converts data from domain $\mathrm{Y}$ to domain $\mathrm{O}$, learns to minimize $\log \left(1-D_{O}(G(y))\right) . F$ and $D_{Y}$ are trained in the same way as $\mathrm{G}$ and $D_{0} . D_{c f_{o} o}$ is a classifier that classifies the opacity $G(y)$ where $D_{c f_{-} o}$ is trained to achieve high classification accuracy.

\subsection{Domain Transformation by Semi-supervised Cycle GAN}

We apply domain transformation to the ROI images created in Section 2.1 using semi-supervised Cycle GAN. As shown in Fig. 3, $Y_{\text {train }}$ and $O_{\text {train }}$ are used to train Cycle GAN, and the domain transformation is performed on $Y_{\text {test }}$ and $O_{\text {test }}$.

As shown in Fig. 4, the structure of the semi-supervised Cycle GAN consists of a combination of the standard Cycle GAN and a trained classifier. The purpose of this model is to learn domain transformation between two domains $\mathrm{Y}$ and $\mathrm{O}$ with given training samples $\left\{y_{i}\right\}_{i=1}^{N}$ 
where $y_{i} \in Y_{\text {train }}$ and $\left\{o_{j}\right\}_{j=1}^{M}$ where $o_{j} \in O_{\text {train }} . N$ and $M$ are the numbers of samples in set $Y_{\text {train }}$ and $O_{\text {train }}$, respectively.

In the standard Cycle GAN, the loss functions Eq. (1) through (3) are used for training $G, F, D_{Y}, D_{O}$.

$$
\begin{aligned}
& L_{G A N}\left(G, D_{O}, Y, O\right) \\
& =E_{o \sim P_{\text {data }}(o)}\left[\log D_{O}(o)\right] \\
& +E_{y \sim P_{\text {data }}(y)}\left[\log \left(1-D_{o}(G(y))\right)\right] \\
& L_{G A N}\left(F, D_{Y}, O, Y\right) \\
& =E_{y \sim P_{\text {data }}(y)}\left[\log D_{Y}(y)\right] \\
& +E_{o \sim P_{\text {data }}(o)}\left[\log \left(1-D_{Y}(F(o))\right)\right] \\
& L_{c y c}(G, F) \\
& =E_{y \sim P_{\text {data }}(y)}[\|F(G(y))-y\|] \\
& +E_{o \sim P_{\text {data }}(o)}[\|G(F(o))-o\|] \\
& L_{\text {identity }}(G, F) \\
& =E_{o \sim P_{\text {data }}(o)}[\|G(o)-o\|] \\
& +E_{y \sim P_{\text {data }}(y)}[\|F(y)-y\|]
\end{aligned}
$$

We denote the data distribution as $y \sim p_{\text {data }}(y)$ and $o \sim p_{\text {data }}(o)$. The domain transformation is learned by minimizing the loss function of Eq. (1), but since this loss function alone will learn to map the same output pattern to any input images, the loss functions Eq. (2) and (3) are introduced. Eq. (2) is called Cycle Consistency Loss, which constrains the original data $y$ and $o$ to match the generated data $F(G(y))$ and $G(F(o))$, respectively. Eq. (3) is called Identity Mapping Loss, which constrains the generator not to convert any data that have belonged to the target domain.

In the semi-supervised Cycle GAN, we introduce a new constraint to re-train Cycle GAN using ResNet. First, we generate (fake) domain $\mathrm{O}$ data $G(y)$ from domain Y by Cycle GAN. ResNet $D_{c f_{-} o}$ trained on domain $\mathrm{O}$ is used to identify the data $G(y)$. The classification results are fed back to the Cycle GAN for re-train. In the re-training, only the ROI images $\left\{y_{k}^{(a n n o)}\right\}_{k=1}^{L}$ are used, where $y_{k}^{(\text {anno })} \in Y_{\text {train_anno }}$ and $Y_{\text {train_anno }}$ contains annotated CT images of five patients per opacity class. L is the number of samples in dataset $Y_{\text {train_anno }}$. For learning $G$ in the semi-supervised Cycle GAN, the loss function shown in Eq. (4) is used in addition to Eqs. (1) through (3).

$$
\begin{aligned}
& L_{\text {opacity }}\left(G, D_{c f_{-} o}\right) \\
& =E_{y^{(a n n o)} \sim P_{\text {data }}\left(y^{(a n n o)}\right)}\left[\log D_{c f_{-} o}\left(G\left(y^{(a n n o)}\right)\right)\right]
\end{aligned}
$$

Our full loss function is:
Table 2. Stride width and the number of ROI images set for each opacity.

\begin{tabular}{l|cc|cc}
\hline \multirow{2}{*}{ Opacity } & \multicolumn{2}{|c|}{$\begin{array}{c}\text { The number of } \\
\text { ROI images }\end{array}$} & \multicolumn{2}{c}{ Width of stride } \\
\cline { 2 - 5 } & Domain Y & Domain O & Domain Y & Domain O \\
\hline CON & 3071 & 3447 & 8 & 11 \\
DN & 3023 & 3311 & 16 & 14 \\
EMP & 3122 & 3021 & 24 & 27 \\
GGO & 3460 & 3273 & 12 & 18 \\
HCM & 3236 & 3434 & 13 & 13 \\
NOR & 3117 & 3035 & 29 & 32 \\
\hline
\end{tabular}

$$
\begin{aligned}
L\left(G, F, D_{A}, D_{B}, D_{c f_{-} o}\right) & =L_{G A N}\left(G, D_{B}, A, B\right) \\
& +L_{G A N}\left(F, D_{A}, B, A\right) \\
& +\lambda_{1} L_{c y c}(G, F) \\
& +\lambda_{2} L_{\text {identity }}(G, F) \\
& +\lambda_{3} L_{\text {opacity }}\left(G, D_{c f_{-} o}\right)
\end{aligned}
$$

where $\lambda_{1}, \lambda_{2}$, and $\lambda_{3}$ are bias terms, which are set to 40,5 , and 0.2 , respectively. $G$ and $F$ are trained to satisfy the objective function expressed by Eq. (6).

$$
G^{*}, F^{*}=\arg \min _{G, F} \max _{D_{Y} D_{O}} L\left(G, F, D_{Y}, D_{O}, D_{c f_{-} o}\right)
$$

Y toO transformation is realized by the above procedure.

\section{Experimental Results}

The effectiveness of the domain transformation YtoO and OtoY is verified using the trained ResNet. In YtoO transformation, after domain $\mathrm{Y}$ data are transformed fake $\mathrm{O}$ by YtoO transformation, they are input to ResNet trained on domain $\mathrm{O}$, and six-class classification is performed. Then, we measure classification performance. The classification performance of the other methods shown in Table 1 is also examined to compare. We perform t-test on the F-measure of the proposed method and other methods to verify whether there are significant differences in terms of $\mathrm{p}$-values.

\subsection{Creating Dataset}

If the stride width of each class is kept constant when generating ROI images, the number of ROI images of each class will be highly biased. Therefore, in this study, we adjusted the stride width for each opacity to keep the number of ROI images as around 3000 for each class. Table 2 shows the stride width and the number of ROI images for each opacity. The generated ROI images have opacity (class) labels based on the annotation by the radiologists. 
Table 3. The number of ROI images used for training and testing. The number of training data for each opacity is about 1000 , and that of testing data is about 2000. The same training data and testing data are used for both YtoO and OtoY transformation.

\begin{tabular}{l|cc|cc}
\hline \multirow{2}{*}{ Opacity } & \multicolumn{2}{|c|}{ Training data } & \multicolumn{2}{c}{ Testing data } \\
\cline { 2 - 5 } & Domain Y & Domain O & Domain Y & Domain O \\
\hline CON & $1022(95)^{* 1}$ & $1027(104)^{* 2}$ & 2049 & 2420 \\
DN & $1018(107)$ & $1020(98)$ & 2005 & 2291 \\
EMP & $1020(105)$ & $962(105)$ & 2102 & 2059 \\
GGO & $989(108)$ & $996(108)$ & 2471 & 2277 \\
HCM & $1003(96)$ & $1021(96)$ & 2233 & 2413 \\
NOR & $1003(105)$ & $1024(101)$ & 2114 & 2011 \\
\hline
\end{tabular}

*1 The values in parentheses are the number of annotated data used in the training of YtoO transformation.

*2 The values in parentheses are the number of annotated data used in the training of OtoY transformation.

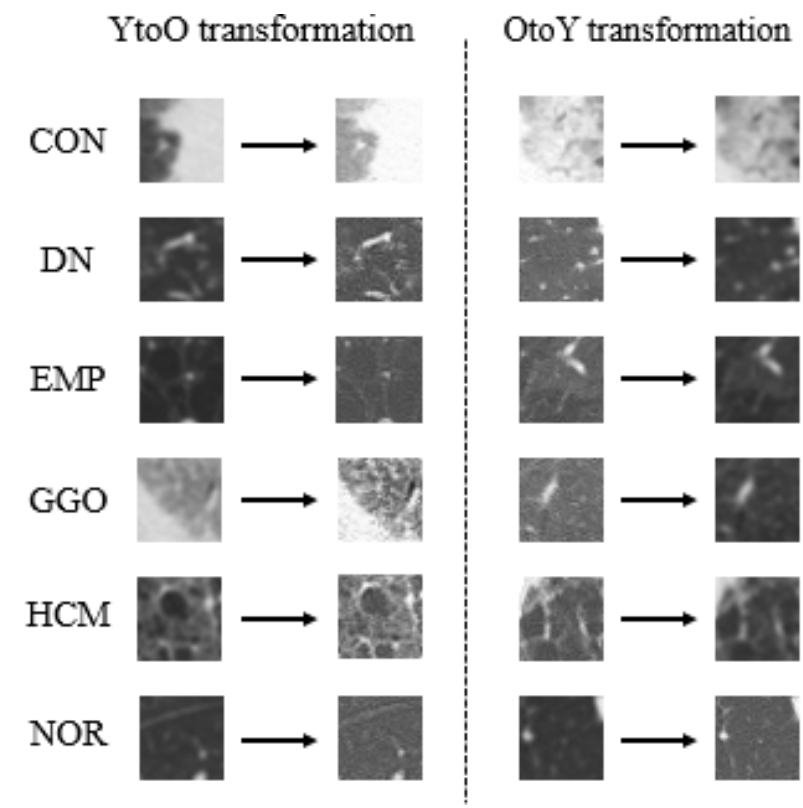

Fig. 5. Example of domain transformation using semi-supervised cycle GAN. The left side shows ROI images after YtoO transformation, and the right side show the one after OtoY transformation.

\subsection{Results of ResNet Training}

We trained ResNet using the generated ROI images. ResNet learned the ROI-based opacity classification for domains $\mathrm{Y}$ and $\mathrm{O}$, respectively. We adopted ResNet34, where the original filter size $7 \times 7$ of the first convolution layer was changed to $3 \times 3$. Then we normalized the value range to $[-1,1]$ as a preprocessing of ROI images used for the training of ResNet. The parameters for training were 20 epochs and mini-batch size 16, and we used Adam $^{5}$ as the optimization method. As a result of the training, the accuracy for the training data in domain $\mathrm{Y}$ was $98.8 \%$, and that for domain $\mathrm{O}$ was $98.1 \%$. The semi-supervised learning of the domain transformations is performed by a model that combines the trained ResNet with a standard Cycle GAN. In the case of YtoO transformation, the ResNet trained on domain $\mathrm{O}$ is incorporated into the Cycle GAN.

\subsection{Results of Domain Transformation by Semi- supervised Cycle GAN}

In this study, the number of residual blocks in the generator was set to 3 because the ROI image size input to the Cycle GAN is small, i.e., 32 x 32 [pixels]. Since the data are grayscale images, the number of input channel is one. Cycle GAN was trained using the training data shown in Table 3. The obtained images before and after the domain transformation using the semisupervised Cycle GAN are shown in Fig. 5.

\subsection{Performance Evaluation}

We measure the classification performance of ResNet on the domain transformed ROI images. In the case of Y toO transformation, ResNet trained with domain $\mathrm{O}$ was used to classify the opacity of the transformed data from domain Y. For the performance evaluation, we used precision, recall, and F-measure. The results averaged over 20 independent trials for YtoO and OtoY transformation are shown in Table 4 and Table 5, respectively. The proposed method shows the best 
Table 4. Precision, recall and F-measure obtained by each method and the results of t-test in the case of YtoO transformation. The values are the average over 20 trials. P-value is calculated by t-test on F-measure between the proposed method and other methods.

\begin{tabular}{lcccc}
\hline Method & Precision (\%) & Recall (\%) & F-measure & p-value \\
\hline No domain transformation & $36.5 \pm 8.6$ & $28.6 \pm 6.4$ & $0.228 \pm 7.8$ & $1.83 \times 10^{-17}$ \\
Cycle GAN & $67.9 \pm 3.6$ & $65.8 \pm 4.2$ & $0.655 \pm 4.5$ & $4.73 \times 10^{-7}$ \\
Method of comparison & $71.3 \pm 2.0$ & $68.4 \pm 2.6$ & $0.682 \pm 2.6$ & $1.13 \times 10^{-7}$ \\
Semi-supervised Cycle GAN & $\mathbf{7 4 . 0} \pm \mathbf{1 . 0}$ & $\mathbf{7 2 . 7 \pm 0 . 9}$ & $\mathbf{0 . 7 2 7} \pm \mathbf{0 . 9}$ & - \\
\hline
\end{tabular}

Table 5. Precision, recall and F-measure obtained by each method and the results of t-test in the case of OtoY transformation. The values are the average over 20 trials. P-value is calculated by t-test on F-measure between the proposed method and other methods.

\begin{tabular}{lcccc}
\hline Method & Precision (\%) & Recall (\%) & F-measure & p-value \\
\hline No domain transformation & $35.0 \pm 4.5$ & $37.0 \pm 2.2$ & $0.319 \pm 1.9$ & $5.80 \times 10^{-42}$ \\
Cycle GAN & $75.2 \pm 1.6$ & $73.4 \pm 1.6$ & $0.730 \pm 2.0$ & $6.00 \times 10^{-3}$ \\
Method of comparison & $73.4 \pm 2.7$ & $70.1 \pm 5.9$ & $0.692 \pm 6.4$ & $1.13 \times 10^{-3}$ \\
Semi-supervised Cycle GAN & $\mathbf{7 6 . 4} \pm \mathbf{1 . 4}$ & $\mathbf{7 4 . 7 \pm 1 . 4}$ & $\mathbf{0 . 7 4 5} \pm \mathbf{1 . 4}$ & - \\
\hline
\end{tabular}

performance for both YtoO and OtoY transformation. Besides, the standard deviation of the results obtained by the proposed method is the smallest, confirming that the classification performance is stable when the ResNet is diverted.

\section{Conclusion}

In this study, we proposed a semi-supervised Cycle GAN that combines a standard Cycle GAN with a trained classifier to perform domain transformation of chest CT images. In the experiment, we classified the normal opacity and five abnormal opacities of diffuse lung diseases in the CT images after the domain transformation and clarified the effectiveness of the proposed method.

\section{Acknowledgments}

This work was supported by JSPS KAKENHI Grant Number JP19K12120.

\section{References}

1. Arnaud Arindra Adiyoso Setio, Francesco Ciompi, Geert Litjens, et al., Pulmonary Nodule Detection in CT Images: False Positive Reduction Using Multi-View Convolutional Networks, IEEE Transactions on Medical Imaging, 2016, pp. 1160-1169

2. Jun-Yan Zhu, Taesung Park, Phillip Isola, Alexei A. Efros, Unpaired Image-to-Image Translation using CycleConsistent Adversarial Networks, International Conference on Computer Vision, 2017
3. Masashi Miyake, Shingo Mabu, Shoji Kido, Takashi Kuremoto, Yasushi Hirano, Domain Transformation of Chest CT Images Using Cycle GAN and Its Application to Classification Systems, The $38^{\text {th }}$ JAMIT Annual Meeting, 2019, pp. 108-115

4. Kaiming He, Xiangyu Zhang, Shaoqing Ren, Jian Sun, Deep Residual Learning for Image Recognition, IEEE Conference on Computer Vision and Pattern Recognition, 2016

5. Diederik P. Kingma, Jimmy Lei Ba, ADAM: A method for stochastic optimization, Proceedings of the 3rd International Conference on Learning Representations, 2015 